\title{
Developing a new quantitative account of backward masking
}

\author{
Gregory Francis* \\ Department of Psychological Sciences, Purdue University, 1364 Psychological Sciences Building, \\ West Lafayette, IN 47907-1364, USA
}

Accepted 15 July 2002

\begin{abstract}
A new general explanation for u-shaped backward masking is analyzed and found to predict shifts in the interstimulus interval (ISI) that produces strongest masking. This predicted shift is then compared to six sets of masking data. The resulting comparisons force the general explanation to make certain assumptions to account for the data. In this way, the experimental data promote the development of a new theory of backward masking. The new theory suggests interpretations of the data that are sometimes novel, often more precise, and sometimes contrary to interpretations that are prevalent in the literature.
\end{abstract}

(c) 2003 Elsevier Science (USA). All rights reserved.

Keywords: Attention; Eccentricity; Metacontrast; Practice

\section{Introduction}

Backward masking refers to impaired performance on some judgment of a target stimulus when it is followed by a mask stimulus. In visual backward masking, both the target and mask stimuli are usually very brief (often less than $50 \mathrm{~ms}$ ). The target stimulus and the observer's task are always designed so that if the target stimulus is presented by itself it is easy for the observer to perform whatever judgment about the

\footnotetext{
${ }^{*}$ This material is based upon work supported by the National Science Foundation under Grant No. 0108905. The author was also partially supported by a fellowship at the Hanse Wissenschaftskolleg, Delmenhorst, Germany.

E-mail address: gfrancis@psych.purdue.edu (G. Francis).
} 
target is required. However, presentation of a mask stimulus, even a $100 \mathrm{~ms}$ after the target has turned off, can make the observer's task of judging something about the target exceedingly difficult. In some cases observers report not seeing the target at all.

Backward masking is a tool that is used throughout experimental psychology to interrupt information processing. If a very strong mask is used then whatever cognitive mechanisms are involved in processing a stimulus (e.g., word recognition) seem to be halted by the appearance of the mask as it obliterates the stimulus representation of the target. As the mask onset is delayed relative to the target onset, more of the target can be processed, and so details about the amount and order of information processing can be deduced. Often times performance on some task is plotted against the interstimulus interval (ISI), or the stimulus onset asynchrony (SOA), between the target and the mask to show some characteristic about the time course of information processing. For this approach to make sense, the performance curve plotted against ISI or SOA (often called a masking function) needs to be monotonic increasing, thereby indicating that as the mask comes later, the target is more fully processed.

Masking functions are not always monotonic increasing. For some situations the masking function is u-shaped (e.g., Alpern, 1953). For short SOAs or ISIs the target is clearly seen, and the required task fairly easy to perform. For middle duration SOAs (often around $80 \mathrm{~ms}$ ), the target is harder to see and the task difficult to perform. For long duration SOAs the task performance is again quite good, perhaps because the target is processed before the mask appears. That going from a short duration SOA to a medium duration SOA should cause a detrimental effect on the observer's task is interesting because it implies that the effect of the mask is not just to halt the processing of the target. If the mask simply halted target processing, then increases in SOA would be expected to allow for more processing and so better performance on the task (or at least, not worse performance). Because of this characteristic, the u-shaped masking function and its properties have been heavily investigated (see Breitmeyer \& Öğmen, 2000 for a recent review).

Experimental data suggest that the difference between monotonic masking functions and $\mathrm{u}$-shaped masking functions are related to the overall strength of masking. Kolers (1962) noted that monotonic masking functions appeared for high energy masks and $\mathrm{u}$-shaped masking functions appeared for masks that were similar in energy to the target. This relationship holds across different types of masks, including pattern masks that have contours overlapping the target (e.g, Hellige, Walsh, Lawrence, \& Prasse, 1979; Spencer \& Shuntich, 1970; Turvey, 1973), metacontrast masks that do not overlap the target (e.g., Schiller, 1965; Weisstein, 1972), and masks consisting of a homogenous field or disk that covers the target (e.g., Stewart \& Purcell, 1974).

Despite its ubiquitous use in cognitive psychology as a means of curtailing information processing, and a large literature describing interactions between the target and mask stimuli under various conditions, the fundamental mechanisms involved in masking are not well understood. Few theories of backward masking are mathematically defined, and of those that are, Francis (2000) showed that they all share a common computational approach. Although this might be taken as a convergence of 
ideas, it also can be interpreted as a failure to recognize that there are viable alternatives. Francis (2000) explored quantitative methods of producing a u-shaped masking function and identified three distinct methods. This paper focuses on one of those methods, called efficient masking. Efficient masking is, in some respects, the simplest of the methods identified by Francis (2000) for producing a u-shaped masking function; and, at least for some systems, it is analytically tractable. This paper begins the development of a theory based on efficient masking. To make the current paper self contained, the following section briefly reviews the properties of efficient masking.

\section{Efficient masking}

Fig. 1 schematizes the hypothesized stages of the theory. The target and mask both contribute to a visual response function (VRF) that is produced by the target stimulus. This VRF feeds into a calculation of a visual percept, which in turn determines behavior on an experimental task. Efficient masking defines quantitative terms for the VRF and the percept calculation.

Label the VRF as $x(t)$, and define $x(t)$ by the following differential equation:

$$
\frac{\mathrm{d} x}{\mathrm{~d} t}=-A x+(B-C x) I(t)-(D+E x) J(t),
$$

where $A, B, C, D$, and $E$ are non-negative parameters, and where $I(t)$ and $J(t)$ correspond to target and mask input signals, respectively. For backward masking conditions, define the target, $I(t)$, and mask, $J(t)$, terms in Eq. (1) as

$$
I(t)= \begin{cases}I & \text { for } 0 \leqslant t<\tau_{1} \\ 0 & \text { otherwise }\end{cases}
$$

and

$$
J(t)= \begin{cases}J & \text { for } \tau_{1}+\tau_{2} \leqslant t<\tau_{1}+\tau_{2}+\tau_{3}, \\ 0 & \text { otherwise }\end{cases}
$$

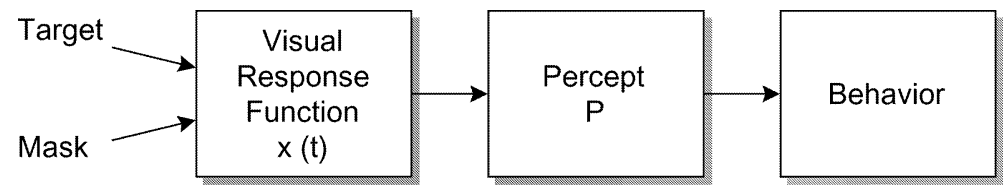

Fig. 1. A box diagram of the system used by Francis (2000) to investigate quantitative approaches to backward masking. The target and mask generate signals that contribute to a visual response function (VRF), which corresponds to the visual system's response to the target. This is represented by a single number, $x(t)$. The VRF feeds into a calculation of the percept of the target, which is given a strength value, $P$. The percept strength then is mapped onto experimentally measured behavior. This mapping depends on the experimental task and the context of the observer. 
Here, time zero corresponds to onset of the target, $\tau_{1}$ corresponds to the duration of the target, $\tau_{2}$ corresponds to the ISI duration between offset of the target and onset of the mask, and $\tau_{3}$ corresponds to the duration of the mask. When the target is present, $x(t)$ grows toward the value $B I /(A+C I)$. During a positive ISI, $x(t)$ undergoes passive decay toward the value zero. When the mask is present, $x(t)$ decreases toward the value $-D J /(A+E J)$. After mask offset, $x(t)$ again decays toward the value zero. With Eqs. (2) and (3), the differential equation in (1) is piecewise linear, and an analytic solution is given in the appendix of Francis (2000).

Eq. (1) is not the only type of system that can use the efficient masking method, however, it has a number of characteristics that motivate its investigation. First, Eq. (1) includes a number of systems that have been used in studies of visual perception for other purposes. For example, in the neural network literature, Eq. (1) is described as a shunting equation and versions of it have been used to explain aspects of brightness perception (Grossberg, 1983), visual persistence (Francis, 1999), and the behavior of cells in the visual system (Gaudiano, 1992). Second, the use of Eq. (1) is similar to the linear systems approach that is popular in studies of early vision (e.g., Loftus, Duncan, \& Gehrig, 1992; Sperling \& Sondhi, 1968). In other uses of this approach, the details of mechanisms are often characterized by their transfer functions, however, the mechanisms can also be characterized by a set of differential equations. A third justification for studying Eq. (1) is a pragmatic one. Because Eq. (1) is a linear differential equation, its analytic solution is known and this allows further investigation to proceed. Although similar (and possibly more interesting behavior) may arise in nonlinear systems, it seems prudent to start by investigating a system that has a known method of analysis. Finally, the behavior of Eq. (1) captures some basic intuitions into what is going on in the visual system. Namely, a target stimulus invokes some kind of response in the visual system that gradually decays away and a mask stimulus can interfere with the target's response. Even if Eq. (1) is incorrect in details, it is worth pursuing how many properties of the backward masking can be understood by this coarse description of the visual system.

As Fig. 1 shows, the VRF contributes to a calculation of percept strength, $P$. Conceptually, this is a single number that gives an indication of the quality of the information acquired by the visual system regarding the target (see Busey \& Loftus, 1994; Loftus et al., 1992). (Presumably, a different and separate measurement would be made for the mask. The percept strength of the mask is not considered here.) The percept strength is measured as an integral of some function of the VRF

$$
P=\int_{0}^{T} F[x(t)] \mathrm{d} t .
$$

Here $F[]$ is a function that clarifies the relationship between the VRF and visual percepts, and $T$ is an upper limit on the integration time. The general hypothesis of this system is that concepts such as percepts and information are meaningfully defined as time integrals of VRFs. Efficient masking supposes that the percept strength is the duration of the VRF above a fixed threshold (other methods of masking 
suppose different computations, see Francis, 2000 for details). The duration of the VRF above a threshold, $G$, is computed by setting

$$
F[x]= \begin{cases}1 & \text { for } x>G, \\ 0 & \text { otherwise. }\end{cases}
$$

As Fig. 1 indicates, values of $P$ should be related to experimental behavior. To be more precise, larger $P$ values should correspond to better perception of the target and its characteristics. The exact mapping between $P$ and experimental behavior likely depends on the details of the experimental task and the characteristics of the observer; the mapping should at least include ceiling and floor effects.

Efficient masking effects explain the downward part of a u-shaped masking function only when the effect of the mask is so weak that the VRF continues to persist even after offset of the mask signal. Francis (2000, Theorem 1) proved that with this requirement satisfied, $P$ is a decreasing function of ISI $\left(\tau_{2}\right)$. When the requirement is not met, $P$ is either an increasing function of ISI or does not change with ISI. The proof is mathematically straightforward, but an analogy is nearly as convincing as the proof, and will be continued throughout this paper.

Suppose you have a cup of coffee that is too hot to drink. You want the coffee to reach a drinkable temperature as quickly as possible, and you can do so by adding a small container of refrigerated cream. Suppose that the cream is not so cold that adding it immediately will bring the coffee to a drinkable temperature. By Newton's law of cooling, the rate of heat transfer between the coffee and the air around it is proportional to the temperature difference across the coffee-air surface. Thus, for constant room temperature, the hotter the coffee, the faster it cools. Adding the cream right away cools the coffee at a time when there would otherwise be a high rate of cooling. After the cream is added the coffee continues to cool, but more slowly than it would have cooled had the cream not been added. Adding the cream a bit later allows the natural rapid cooling of the coffee to occur unperturbed and then still allows for the direct cooling effect of the cream (Gardner, 1961, p. 145). The most efficient use of the cream is to add it after an appropriate delay so that the coffee has cooled to a temperature where just as you add the cream, the coffee temperature becomes drinkable.

The same behavior is the basis for efficient masking and the u-shaped masking function. The time it takes for the coffee to cool to a drinkable temperature is analogous to the duration of the target VRF being above a threshold (this duration corresponds to the target's percept). A goal of getting the coffee to a desired temperature as quickly as possible is analogous to a goal of having maximum masking for a given mask. The temperature of the coffee is analogous to the VRF produced by the target. The cooling effect of the cream is analogous to the effect the mask has on the target's VRF.

The properties of efficient masking explain why the percept of the target decreases with later presentation of the mask. This is the decreasing part of the u-shaped masking function. The increasing part of the u-shaped masking function occurs because with long SOAs the mask arrives so late that it has a diminished impact on the target percept.

No one has yet proposed a specific model of backward masking that utilizes the properties of efficient masking to produce a u-shaped masking function. This paper 
begins the development of such a model by exploring some of the basic properties of efficient masking and relating those properties to experimental data. The development begins by identifying general properties of efficient masking that are likely to exist in any efficient masking based model, provided that model does not include specific mechanisms that counteract the basic efficient masking properties. Once the basic mechanisms of efficient masking are identified, they can be used to develop a model in two ways. First, experimental data can be interpreted in terms of the basic mechanisms of efficient masking. This promotes model development by identifying how different experimental conditions correspond to different components of the model. Second, experimental data that are inconsistent with the basic mechanisms of efficient masking can be identified. This promotes model development by forcing the creation of specific mechanisms for the model that counteract the basic mechanisms of efficient masking. Thus, the goal of this paper is not to test and possibly reject the efficient masking approach, but rather to explore what type of model would be needed to use the efficient masking approach to account for experimental data. Subsequent to this investigation, it may well be reasonable to conclude that the assumptions needed to make the model match the data are invalid. If that is the conclusion, then the efficient masking approach could be rejected.

Section 3 identifies some of the basic mechanisms of efficient masking. Section 4 then analyzes previously published data on backward masking to connect model mechanisms to experimental conditions. The result is a (partial) model that provides novel interpretations of some experimental data and makes testable predictions.

\section{Properties of efficient masking}

The analysis of efficient masking properties is separated into four parts: effects of the mask input, effects of the target input, effects of the mapping between percept strength and behavior, and combinations of effects. In every case, the analysis focuses on changes in the properties of the u-shaped masking function because it turns out that efficient masking makes particular claims about what contributes to the shape of the masking function.

Francis (2000, Lemma 1) noted that with Eqs. (1)-(5), the ISI that gives rise to the strongest masking (the bottom of a u-shaped masking function), designated $\tau_{2}^{*}$, is calculated as

$$
\tau_{2}^{*}=\frac{1}{A}\left[\ln \left\{\frac{B I\left(1-\mathrm{e}^{-(A+C I) \tau_{1}}\right)}{A+C I}\right\}-\ln \left\{G+\frac{D J\left(1-\mathrm{e}^{-(A+E J) \tau_{3}}\right)}{A+E J}\right\}-(A+E J) \tau_{3}\right] .
$$

In relation to the coffee cooling analogy. $\tau_{2}^{*}$ is the optimal time delay between pouring the coffee into a cup and adding the cream. It is optimal in that the coffee will reach the desired drinkable temperature most quickly. This time can be computed backwards from the desired drinkable temperature. Add the cream at just the moment so that after the cream has mixed with the coffee (and cooled it) the coffee is 
drinkable. Adding the cream sooner makes the natural cooling of the coffee less efficient. Adding the cream later is wasting time, because the cream could have been added sooner.

\subsection{Effects of the mask input}

The ISI for maximal masking can be shifted to larger or smaller values by changing properties of the mask. For example, Francis (2000, Lemmas $2 \& 3$ ) noted that increases in the mask intensity, $J$, or mask duration, $\tau_{3}$, lead to decreases in $\tau_{2}^{*}$. That is, as mask intensity and duration increase, the bottom of the masking curve shifts to smaller ISIs. In relation to the coffee-cooling analogy, if you have colder cream, or more cream (which takes longer to pour), you do not need to wait as long for the coffee to cool before adding the cream brings the coffee to the desired temperature. Fig. 2 shows representative masking curves generated by Eqs. (1)-(5) for different mask durations and mask intensities. For masks that are intense enough and of long enough duration, the strongest mask is at ISI equal zero, and the masking curve is monotonic increasing.

Experimental data (e.g., Bernstein \& Fisicaro, 1977; Breitmeyer, 1978; Spencer \& Shuntich, 1970; Stewart \& Purcell, 1974) generally support the property that ushaped masking curves are more common with weaker masks, and that the ISI for maximal masking shifts to smaller values as mask intensity or duration increases.

\subsection{Effects of the target input}

Lemmas A.1 and A.2 in Appendix A of this paper describe the effect of target intensity, $I$, and target duration, $\tau_{1}$, on the ISI for maximal masking. In efficient masking, increases in target intensity or duration tend to shift the ISI for maximal masking to larger values. That is, the mask must appear later to have its maximum effect. (However, Corollary A.1 shows that the effects saturate.) With regard to the coffee cooling analogy, the target stimulus is like a source of heat that raises the temperature of the coffee. As more heat energy is applied to the coffee (through a hotter burner or longer heating) then the coffee will take longer to cool when the heat source is removed. With hotter coffee, you should wait longer to add the cream, so that the coffee has had sufficient time to cool enough for the cream to bring the coffee to the desired temperature. Fig. 3 shows representative masking curves generated by Eqs. (1)-(5) for different target durations and target intensities.

\subsection{Effects of the mapping between percept strength and behavior}

If the mapping between values of percept strength and behavioral responses changes, the masking curve can be shifted up and down. However, as long as the mapping retains an ordinal relationship between percept strength and behavioral response, the bottom of the masking function will not shift left or right. In efficient masking, the computed percept strength determines the shape of the masking function, so the bottom of the masking curve (excluding ceiling and floor effects) should 

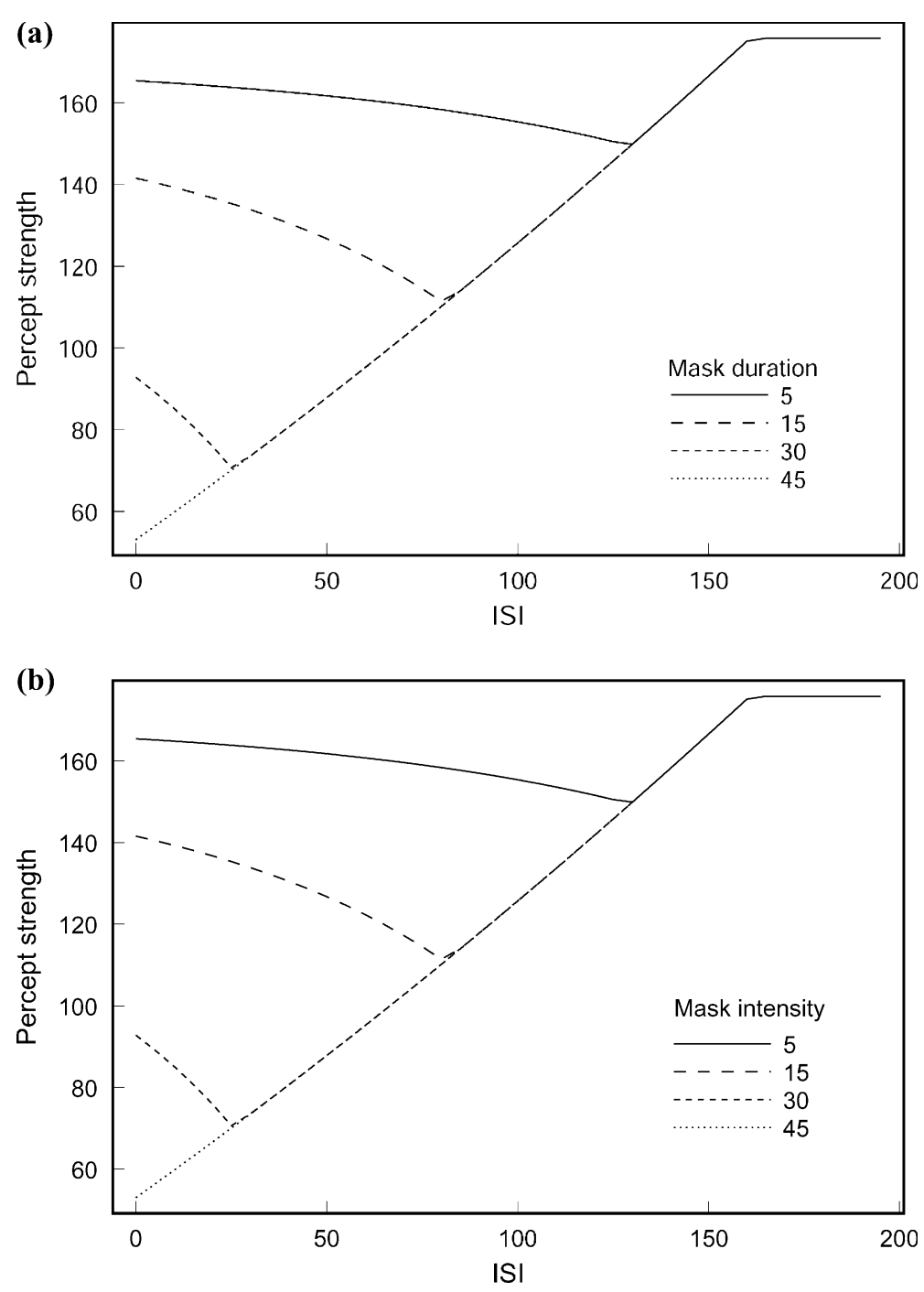

Fig. 2. The effect of varying mask duration and mask intensity on the calculation of percept strength using Eqs. (1)-(6). As mask duration (a) or mask intensity (b) increases, percept strength becomes smaller and the bottom of the u-shaped function shifts to smaller ISI values. For very long mask durations or mask intensities, the pattern of percept strength is monotonic increasing with ISI.

be unchanged across conditions that vary only the mapping of percept strength to behavior. Fig. 4 schematizes these types of interactions and the resulting masking functions that would be generated.

Fig. 4a plots the values of $P$ that are computed for various ISIs using Eqs. (1)-(5). A specific value of $P$ should be related to a certain level of performance on an experimental task. The dashed lines on the plot indicate hypothetical boundaries of $P$ val- 

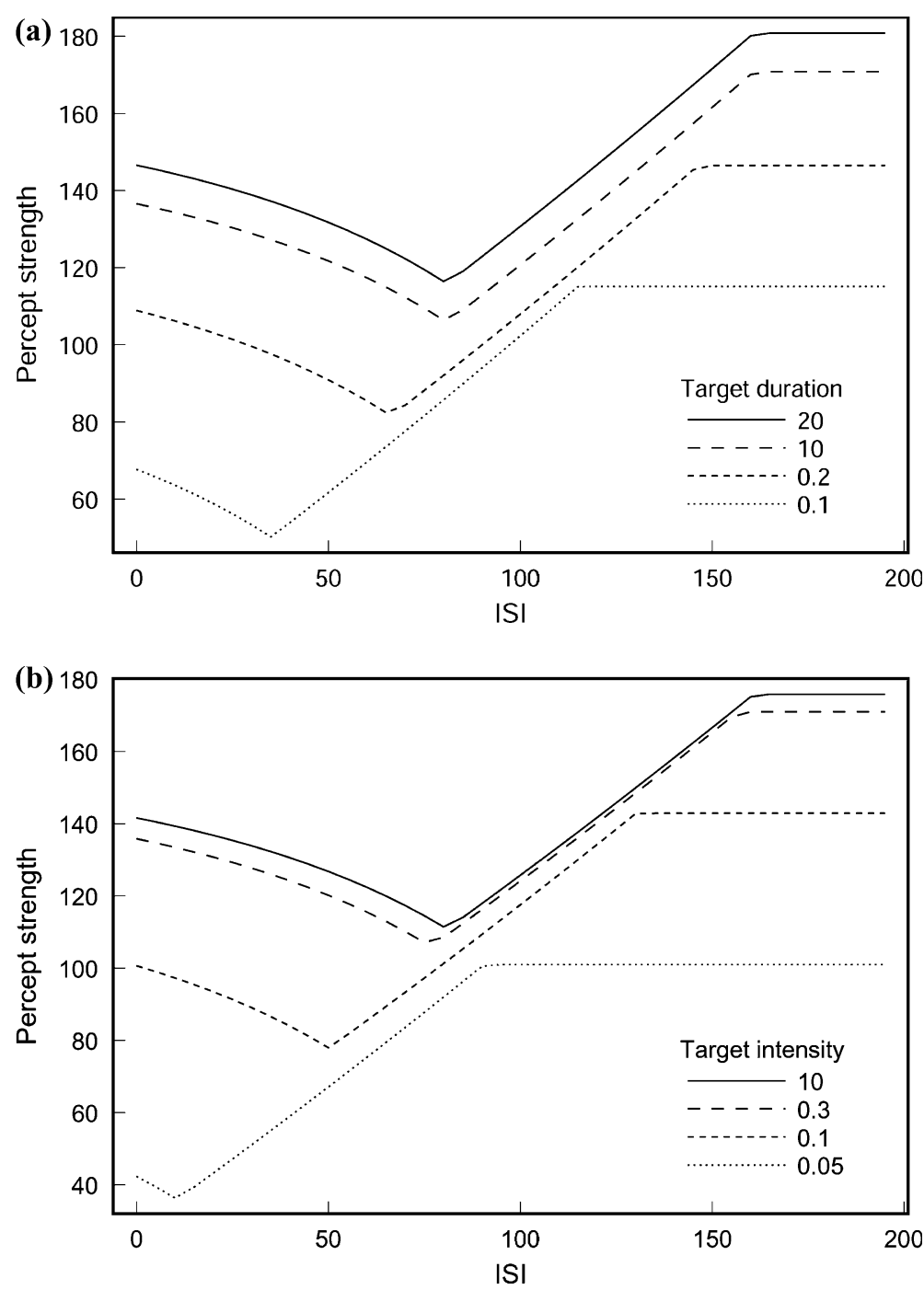

Fig. 3. The effect of varying target duration and target intensity on the calculation of percept strength using Eqs. (1)-(6). As target duration (a) or target intensity (b) increases, percept strength becomes larger and the bottom of the $\mathrm{u}$-shaped function shifts to larger ISI values. For both target duration and target intensity, the shift in the bottom of the u-shaped curve approaches a limit. For target intensity, the increase in percept strength also saturates.

ues that map to floor and ceiling effects in a experimental task. For the line marked "Ceiling easy," any $P$ value greater than 120 would be at ceiling (e.g., $100 \%$ correct in a target identification task). Likewise any $P$ value below 80 (marked "Floor easy") would give rise to a behavioral floor response (e.g., guessing). The solid line in Fig. 4b plots a hypothetical masking function that assumes that percent correct is linearly related to the relative position of $P$ between the corresponding floor and ceiling limits. 

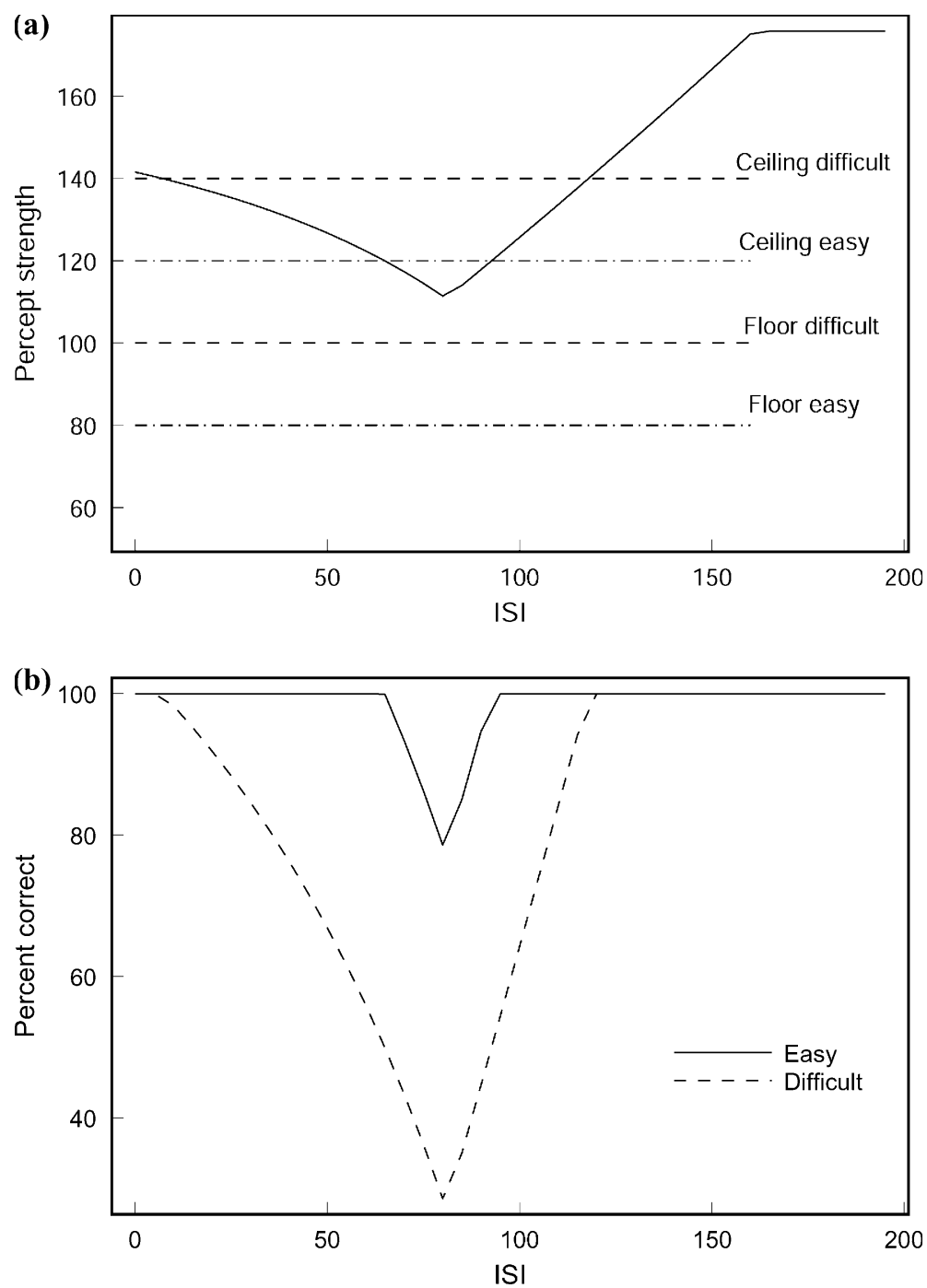

Fig. 4. The effect of changing hypothetical ceiling and floor thresholds that map percept strength to behavioral data. (a) Shows a u-shaped curve relating percept strength to ISI using Eqs. (1)-(6). The dashed lines indicate thresholds for an easy and a difficult task. Percept strength above a ceiling is assumed to lead to perfect performance on the experimental task. Percept strength below a floor is assumed to lead to guessing performance on the experimental task. The ceiling and floor thresholds of the hypothetical difficult task are larger than the ceiling and floor thresholds of the easy task. This indicates that for the difficult task, larger percept strengths are required to reach a given performance level in the experiment. (b) $\mathrm{Hy}$ pothetical masking curves that would be generated by corresponding ceiling and floor thresholds. The easy task shows weaker masking, but the ISI that produces the strongest masking is unchanged across the conditions. 
The lines marked "Ceiling hard" and "Floor hard" in Fig. 4a correspond to boundaries for a masking situation that produces the same $P$ values, but is otherwise more difficult (e.g., there is a secondary task, or more specific information about the target is required). The dashed line in Fig. 4b shows a corresponding masking function. The key observation is that although masking is stronger (measured by smaller percent correct values) for the hard boundaries than for the easy boundaries, the ISI that produces the strongest masking is unchanged.

\subsection{Combinations of effects}

If properties of the mask input, the target input, and the mapping of percept to behavior change simultaneously, the masking functions can vary in almost any manner. Significantly, certain changes in the shape of masking functions can only be produced by combinations of effects.

For example, a characteristic of efficient masking is that if only one of the above effects (target input, mask input, or mapping of percept to behavior) is allowed to vary, then the bottom of the u-shaped masking function cannot shift to the right as the behavioral measure of masking increases. (See Figs. 2-4.) A shift in the masking function down (indicating stronger masking) and to the right would only be possible by a combination of effects among the mask, target, and the mapping between the percept strength and behavior. Fig. 5 shows an example of how this could occur. In the simulations, the mask was either weak (a duration of 15 time units) or strong (a duration of 30 time units). The strong mask produces weaker target percept strengths for shorter ISIs, as shown in Fig. 5a. Simultaneously, the mapping between percept strength and behavior was varied. For the strong mask, any percept strength values below 40 (floor easy) were assumed to be at chance levels of behavioral performance and any percept strength values above 95 (ceiling easy) were assumed to be at perfect performance levels. On the other hand for the weak mask, any percept strength values below 100 (floor difficult) were assumed to be at chance levels of behavioral performance and any percept strength values above 160 (ceiling difficult) were assumed to be at perfect performance levels. The boundaries of performance are indicated by the horizontal lines in Fig. 5a.

Conceptually, the situation is that the weak mask produces a smaller effect on the percept strength, but simultaneously, it is relatively difficult to get percept strength values above the floor threshold. For the strong mask, the factors are switched. The strong mask produces a big effect on the percept strength, but it is relatively easy to get percept strength values above the floor threshold. The result of these effects is shown in Fig. 5b. The masking functions show the separate influences of the mask duration and the mapping of percept strength to behavior. That the bottom of the masking function shifts to a smaller ISI for the strong mask indicates the underlying shape of the percept strength function generated by the different masks, as seen in Fig. 5a. That the masking function generated by the strong mask has weaker overall masking reflects the fact that the strong mask is also put in a context where relatively small percept strength values are mapped onto high behavioral performance values. 

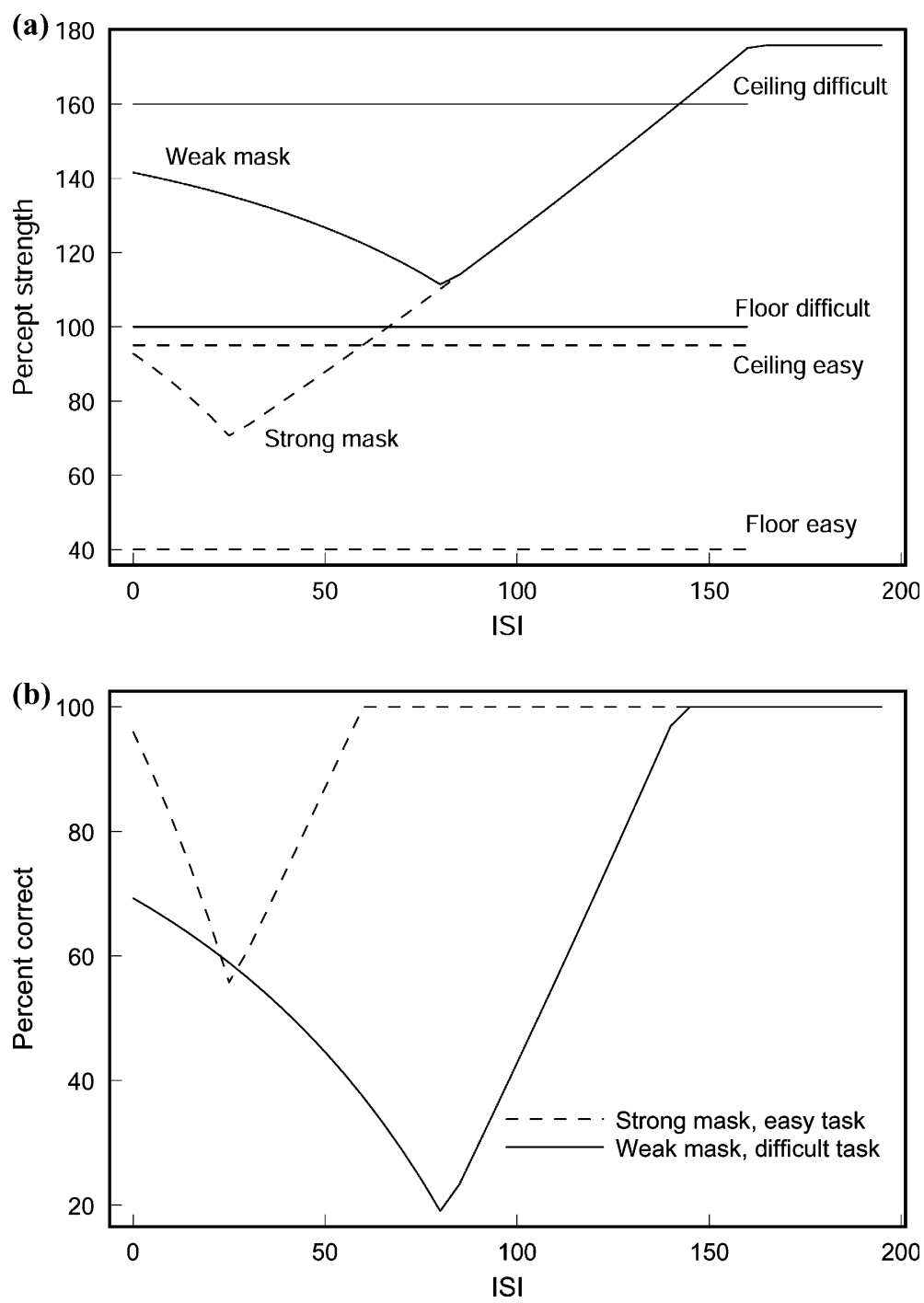

Fig. 5. The combined effect of changing mask duration and the hypothetical ceiling and floor thresholds that map percept strength to behavioral data. (a) Shows two u-shaped curves relating percept strength to ISI. The lower curve corresponds to the stronger (longer duration) mask. The horizontal lines indicate thresholds for an easy and a difficult task. The curve with the weaker mask is associated with the difficult thresholds and the curve with the stronger mask is associated with the easy thresholds. (b) Hypothetical masking curves that would be generated by corresponding conditions. The easy task shows generally weaker masking than the difficult task. However, since the easy task is paired with the strong mask, the bottom of its masking function is shifted to a smaller ISI than the masking function for the difficult task, which is paired with the weak mask. 
Thus, the mapping of percept strength to behavior can overcome the tendency for the strong mask to exhibit stronger masking, but changing the mapping cannot change the location of the bottom of the masking function. The resulting masking functions demonstrate both effects.

\subsection{Conclusions of analysis}

Changes in the mask input, the target input, or the mapping of percept strength to behavior give rise to systematic variations in the ISI that produces the strongest masking of a $\mathrm{u}$-shaped masking function. In fact, since there are so few mechanisms in the simplest efficient masking systems, one can work backwards and use the shape of the masking functions to identify what factors must have been varied. If the experimentally measured magnitude of masking increases and the bottom of the u-shaped masking function shifts to smaller ISIs, efficient masking most naturally interprets this as either due to an increase in the mask signal intensity or duration or due to a decrease in the target signal intensity or duration. On the other hand, if the magnitude of masking increases and the bottom of the $\mathrm{u}$-shaped masking function remains at an unchanged ISI, efficient masking most naturally interprets this as a change in the mapping of percept strength to behavior. Finally, if the magnitude of masking increases and the bottom of the $\mathrm{u}$-shaped masking function shifts to larger ISIs, then efficient masking interprets this as due to a combination of effects due to variation in the target signal, the mask signal, and the mapping between percept strength and behavior.

\section{Model interpretations of experimental data}

We can use the above analysis to begin to create a model that uses the properties of efficient masking. We do this by looking at existing experimental data that measured masking functions across varying ISIs or SOAs. We can note changes in the independent variables in these experiments and look for corresponding shifts in the bottom of the masking functions. We can then hypothesize how an efficient masking model would interpret the effect of the changes in the independent variables.

Although the description of Eqs. (1)-(5) describe $I(t)$ and $J(t)$ as corresponding to the target and mask, we need not limit ourselves to such a strict interpretation. It is equally valid to imagine that the $I(t)$ and $J(t)$ properties correspond not to the physical properties of the target and mask stimuli but instead correspond to responses to those stimuli that are generated by lower levels of the visual system. Such responses can be modified by a variety of environmental and observer properties.

Because efficient masking is defined at a fairly abstract level, changes in experimental variables have very few mechanisms for affecting measured behavior; they can affect only the mask input, the target input, and/or the mapping between percept strength and behavior. Whether masking properties can be characterized at such an abstract level is unknown and is, in part, a topic of this paper. Toward that end, we consider a variety of experimental data and note how a model based on efficient masking would account for the data. 
The following sections consider experimental data that show an increase in the strength of masking and either: do not show a shift in the bottom of the masking function, show a shift to smaller ISI or SOA values, or show a shift to larger ISI or SOA values. Six sets of data are considered that each systematically vary or control for effects of: practice, eccentricity, light adaptation, flicker adaptation, attention, and a word superiority effect. The experimental data are grouped according to how a model based on efficient masking interprets the data.

\subsection{Changes in masking strength with no shift in ISIS}

This section considers data sets that exhibit variations in the overall strength of masking but that have no corresponding shifts in the bottom of the masking function. A model that uses efficient masking interprets this finding as evidence that the experimental manipulation that underlies the change in masking strength influences the mapping between percept strength and behavioral responses (e.g., changes in ceiling or floor effects). The model would not hypothesize that the experimental manipulation that underlies the change in masking strength is related to changes in the intensity or duration of the target or mask signals, because then efficient masking would expect shifts in the ISI or SOA that produces maximal masking.

\subsubsection{Practice}

Hogben and Di Lollo (1984) explored practice effects in backward masking. The target stimulus consisted of a $3 \times 3$ matrix of dots, with one dot missing. The observer's task was to identify the location of the missing dot. The mask stimulus consisted of either a pair of flanking dot matrices or a single dot matrix on one side of the target matrix. The measure of masking was decreases in the percentage correct identifications of the location of the missing dot. Practice increased the percentage correct averaged across observers, which indicates weaker masking with practice. Fig. 6 shows masking functions for different blocks of trials. The data are from the second experiment of Hogben and Di Lollo (1984), which partially controlled for forward masking effects that the target matrix sometimes had on the dots in the mask matrix. In the first experiment of Hogben and Di Lollo (1984), these forward masking effects could sometimes act as cues to indicate to observers where the missing dot was in the target matrix. In the second experiment, one randomly chosen dot in the mask was missing, which interfered with the salience of the cue that was used by observers in the first experiment.

Performance generally gets better with practice, however, there is no shift in the bottom of the masking curve. Across all blocks it remains constant at an SOA of $80 \mathrm{~ms}$. Similar results were found in the first experiment of Hogben and Di Lollo (1984), but there was greater variability in the data and the bottoms of the masking functions were less well defined.

Hogben and Di Lollo (1984) accounted for their data by suggesting that practice resulted in changes in criterion content; primarily as a matter of learning to use the forward masking cue that was quite effective in their first experiment, but less so in the second experiment. This explanation can be interpreted as a more specific instan- 


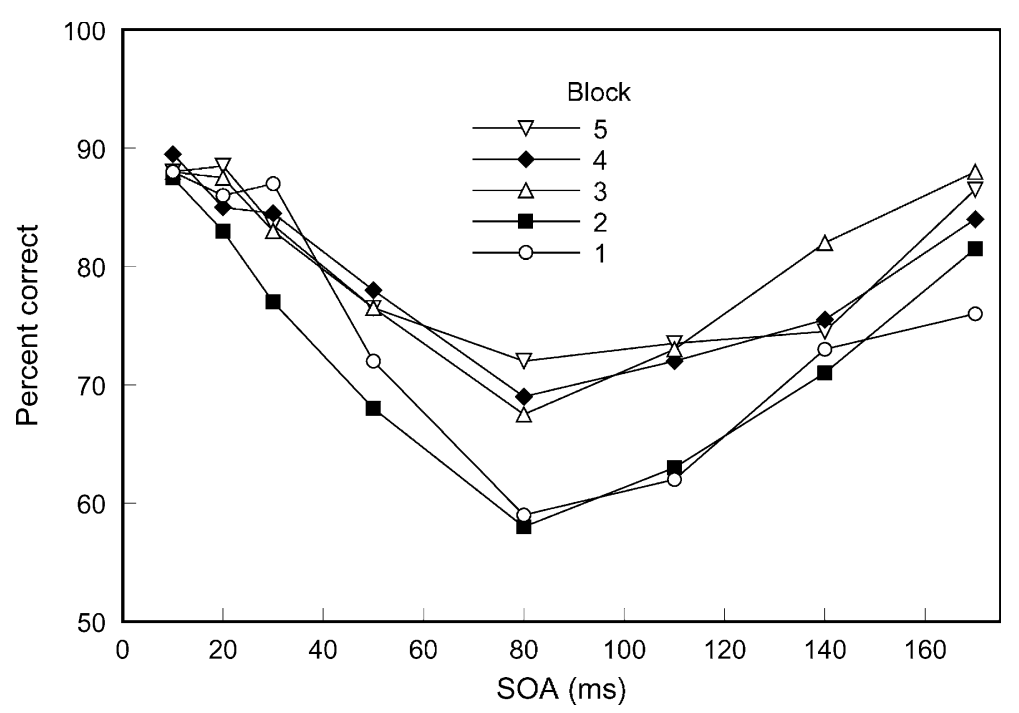

Fig. 6. Experimental data that demonstrate how masking effects weaken with practice. The SOA for maximum masking (the bottom of each masking curve) does not vary across blocks. (Reproduced from a figure in Hogben \& Di Lollo (1984).)

tiation of the explanation within a model that uses efficient masking. Regardless of the specifics of the mechanisms, an interpretation of the data with efficient masking does not allow for the possibility that practice somehow weakens the mask signal or strengthens the target signal that feeds into the computation of the percept. Such changes would have introduced a shift in the bottom of the masking functions. It would be fruitful to explore practice effects in other backward masking paradigms to see if the masking function ever shifts as a result of practice. For example, one might expect that practice would result in perceptual learning, which would enhance the target signal relative to noise (e.g., Dosher \& Lu, 1998; Gold, Bennett, \& Sekuler, 1999) and thereby lead to a shift in the bottom of the masking function to the right.

\subsubsection{Eccentricity}

A common finding is that backward masking is stronger when the stimuli appear in the periphery rather than in the fovea. Although several studies have noted this effect (e.g., Bridgeman \& Leff, 1979; Stewart \& Purcell, 1970), we focus on a study by Stewart and Purcell (1974) because it used a finer grained masking function, with smaller separation between SOAs, than the other experiments. The target was a small rectangle that was sometimes presented for $20 \mathrm{~ms}$ in the middle of a screen. The observer's task was to report whether the target was present or not during a trial. The mask was a three degree square flash of light that followed the target by a variable SOA. In different sessions, the observer was asked to fixate at different points. All fixation points were on the screen for all trials. The fixation points consisted of five dots. Four dots were along the major compass points around the loca- 
tion of the target stimulus. Center fixation was achieved by looking at the middle of the diamond formed by these fixation dots. Fixation $0.6^{\circ}$ left of the target was achieved by asking observers to fixate on the dot to the left of the target location. Fixation $1.7^{\circ}$ left of the target was achieved by asking observers to fixate on a fifth fixation dot, located left of the diamond.

To accommodate differences among observers, Stewart and Purcell (1974) created a unique set of SOAs for each observer. In a pre-test, the SOA for maximum masking was found for center fixation. This SOA was then used to define a set of six SOAs that were multiples of the previously identified best SOA for each observer. The multiplying factors were $0,0.5,1.0,1.5,2.0$, and 2.5. Thus, an SOA of zero and the SOA for maximum masking for the center fixation condition were always included. Fig. 7 shows the results from Stewart and Purcell (1974) by plotting the percentage of correct detections against the order of the SOAs (ranged from smallest to largest), with separate curves for each eccentricity. There is a strong effect of eccentricity. When the point of fixation is farther away detection of the target is poorer. Moreover, the SOA that produces the strongest masking is unchanged with the point of fixation.

The last finding deserves special attention. For the center fixation condition, the found masking curve was expected by the definition of the SOAs. The third SOA is the one that was identified by the pre-experiment as the SOA that produced the strongest masking for a particular observer. Thus, the curve for the center fixation is simply a verification of the pre-test. However, for the $0.6^{\circ}$ and $1.7^{\circ}$ fixation conditions, there was no a priori reason to expect the third SOA would produce the strongest masking. That the SOA for strongest masking is unchanged is interpreted

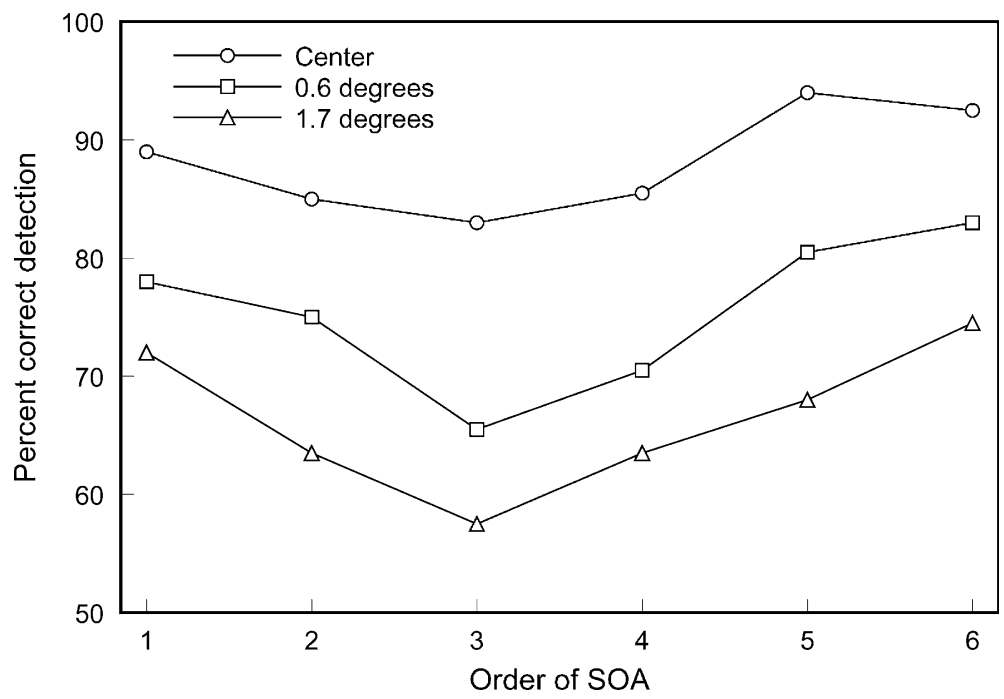

Fig. 7. Experimental data that demonstrate how masking effects increase with eccentricity of the target. The SOA for maximum masking (the bottom of each masking curve) does not vary with distance between the fixation point and the target location. (Reproduced from a figure in Stewart \& Purcell, 1974.) 
by an efficient masking model as evidence that changes in eccentricity correspond to changes in the mapping of percept strength to behavior. Apparently, for more eccentric positions of the target, the percept strength must be larger to promote detection of the target.

This is an interesting conclusion because stronger masking with increased eccentricity has been previously proposed to be related to the presence of stronger (or more) transient signals (e.g., Breitmeyer \& Ganz, 1976). However, in an efficient masking system, the existence of stronger mask signals (transient or not) should cause a shift in the SOA for maximum masking to smaller values. There is no such shift in the data, so the hypothesis of stronger mask signals in the periphery cannot be used in a model based on efficient masking.

We can propose a possible specific mechanism for the general functional observation proposed by efficient masking. It is well known that visual acuity is poorer in the periphery (e.g., Anstis, 1974). Perhaps poorer detection in the periphery with backward masking simply reflects that basic property of visual perception. Significantly then, efficient masking would make the claim that the locus of the periphery effect is not in the strength of signals generated by target or mask stimuli, but in the interpretation of the VRFs engendered by those stimuli. This prediction should allow for comparison of eccentricity effects in a variety of masking and non-masking situations. If the efficient masking explanation is correct, the eccentricity effect should be similar across different experimental paradigms.

\subsection{Increases in masking strength with shifts to smaller ISIs}

This section considers data sets that exhibit increases in the overall strength of masking and have corresponding shifts in the bottom of the masking function to smaller ISIs or SOAs. A model that uses efficient masking interprets this finding as evidence that the experimental manipulation that underlies the change in masking strength either is increasing the intensity or the duration of the mask signal or is decreasing the intensity or the duration of the target signal. Changes in the mapping of percept strength to behavior could also occur, but cannot account for the shift in the ISI that produces maximal masking.

\subsubsection{Light adaptation}

Purcell, Stewart, and Brunner (1974) investigated variations in metacontrast masking (backward masking where the target and mask foreground do not overlap) when observers were light or dark adapted. The task was to identify the location of a black disk that was presented on a bright background in one of two locations. The mask consisted of two black annuli surrounding the possible locations of the target disk. In separate sessions, an observer was adapted for $10 \mathrm{~min}$ to either a light (40 fL) or a dark field. Fig. 8 shows how percent correct detection varied with SOA for light and dark adaptation conditions. The study also explored variations in the illumination of the target background, and Fig. 8 shows only masking curves for a fixed target background illumination. Other target background illuminations showed similar shifts in the masking curve when there were u-shaped masking functions. 


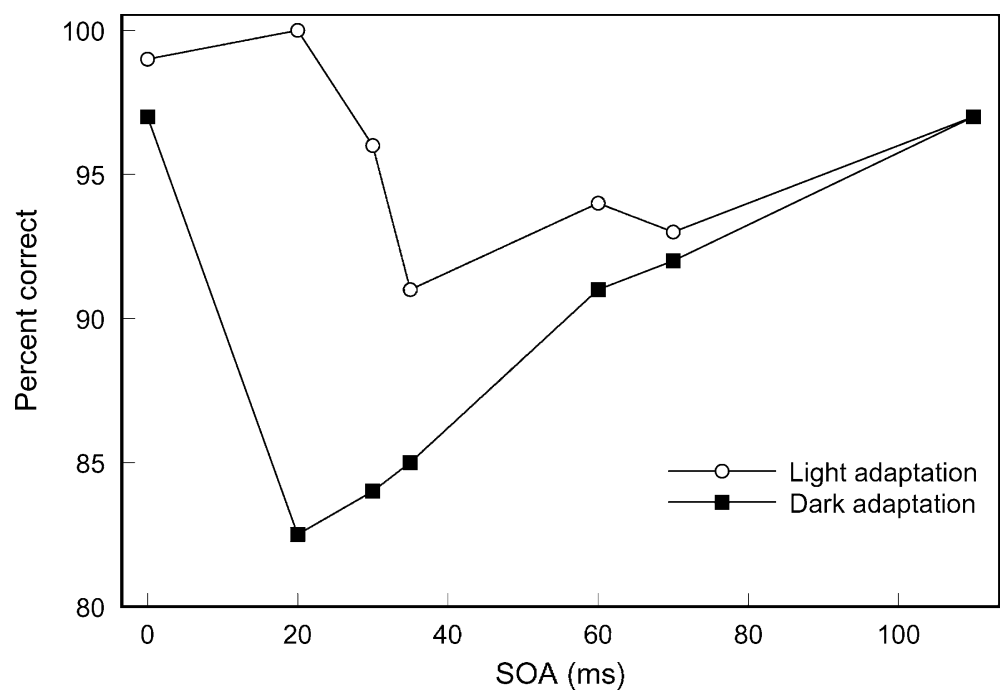

Fig. 8. Experimental data that demonstrate effects of light and dark adaptation on masking functions. Masking is stronger in the dark adapted condition. Moreover, the SOA for maximal masking (the bottom of each curve) is shifted to smaller values for the dark adapted condition. (Reproduced from figures in Purcell et al., 1974.)

As Purcell et al. (1974) noted, masking is generally stronger for dark adapted than light adapted conditions. Moreover, the u-shaped masking function generated under a dark adapted condition has a bottom that is shifted to a smaller SOA relative to the u-shaped masking function in the light adapted condition.

In efficient masking, this pattern of results indicates that the mask signal is stronger in the dark adapted than the light adapted condition. The stronger mask signal can both lead to poorer performance on the experimental task and cause the bottom of the masking curve to be shifted to a smaller SOA (Fig. 2b). Presumably, the mask signal is stronger because dark adaptation makes the visual system more responsive to the black on white contrast of the mask field. Of course, the same increased responsiveness would occur for the target signal as well. An increase in target intensity would be expected to shift the SOA for maximum masking to the right (Fig. 3b). However, because of the saturation effects noted in the corollary to Lemmas A.1 and A.2 (see Appendix A), the shifting due to an increase in target input will sometimes be overwhelmed by larger shifts due to the increase in the mask input. The reverse could also be true in other situations and for different parameters of the system. Consistent with this possibility, Bischof and Di Lollo (1995) found that metacontrast masking was stronger under light adapted than dark adapted conditions. It is not clear that efficient masking predicts the effects of light adaptation in the differing conditions of these experiments, most likely a specific model will need to be created to account for the details. However, efficient masking does allow for varying effects of light adaptation, depending on the stimuli and the parameters of the system. 


\subsubsection{Flicker adaptation}

Perhaps the currently dominant theory of u-shaped backward masking functions is one proposed by Breitmeyer and Ganz (1976) and subsequently elaborated by Breitmeyer (1984). This theory proposed that u-shaped backward masking functions were largely the result of differences in response times by sustained and transient channels. The transient channels respond more quickly than the sustained channels; and the transient channels inhibit the sustained channels, with the latter giving rise to the target percept. In this theory, the mask needs to be delayed relative to the target in order for the inhibitory transient signal to overlap most fully with the slower sustained signal.

One of the interesting characteristics of efficient masking is that it does not require that responses to the target and mask be differentially delayed (neither does it necessarily prohibit such delays). Thus, efficient masking seems an interesting alternative to the transient-sustained theory. However, there is substantial evidence that transient components of the mask are important for backward masking. Thus, it may be fruitful to explore how efficient masking might accommodate some data that demonstrate a transient component.

We focus on a study by Petry, Grigonis, and Reichert (1979), which showed that pre-adaptation to a flicker stimulus could reduce subsequent masking. The target stimulus was a vertical bar, and the mask a pair of flanking vertical bars. The observer's task was to judge the brightness of the target with a magnitude estimation procedure. In one condition, the observer simply saw the target-mask sequence at varying SOAs. In another condition, the observer pre-adapted to a static presentation of the mask stimulus for $10 \mathrm{~s}$ and then saw the target-mask sequence. In three other conditions, the mask presented during the $10 \mathrm{~s}$ pre-adaptation period flashed on and off with one of three off-times. The results of this study are presented in Fig. 9 for one observer, with separate curves for the different conditions. The numbers on the $y$-axis are the magnitudes assigned by the observer, and the intersection of the $x$ - and $y$-axes corresponds to the brightness magnitude assigned by the observer to the background. Smaller numbers indicate stronger masking.

Masking was stronger for the no adaptation and the adaptation without flicker conditions than for any of the conditions with flicker adaptation. Petry et al. (1979) interpreted this as being the result of the pre-adaptation flicker weakening the responsiveness of the transient channel and thereby producing a weaker mask signal. A model based on efficient masking can make the same interpretation, and consistent with this interpretation, the SOA for maximal masking has shifted to the left for the stronger masking conditions. Since Petry et al. (1979) found that adaptation to a transient results in a weakening of the mask signal, this indicates that a model based on efficient masking needs to include a transient component to the mask signal. Additional details of the model need to describe how adaptation to the flicker stimulus can adapt out that transient component.

\subsubsection{Attention}

Recent studies of masking have identified that attention can substantially modulate the effectiveness of a mask. For example, Ramachandran and Cobb (1995) noted that 


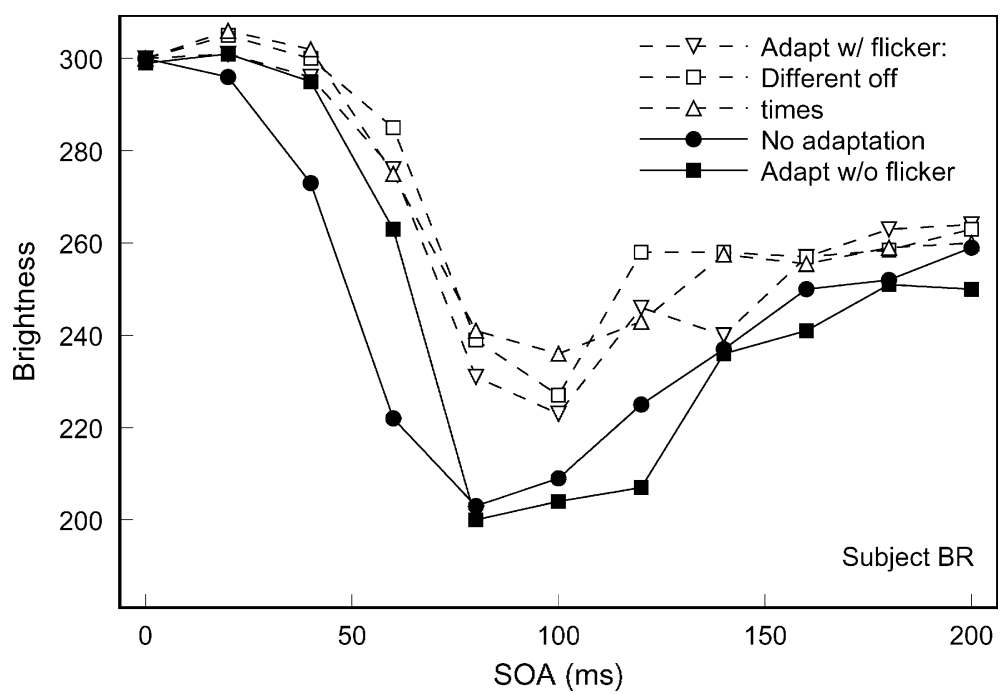

Fig. 9. Experimental data that demonstrate effects of flicker adaptation on masking functions. For each of the flicker conditions (dashed lines) masking is weaker and the SOA for strongest masking (bottom of each curve) is shifted to a larger value. (Reproduced from a figure in Petry et al., 1979.)

a target dot that was strongly masked when in isolation was not masked as well when it was perceived to be grouped with other dots (also see Werner, 1935). Likewise, Shelley-Tremblay and Mack (1999) noted that meaningful stimuli are less susceptible to masking and are stronger maskers. Enns and Di Lollo (1997) have also noted that some masking effects seem to depend on attention not being focused on the target.

A study by Weisstein (1966) varied stimulus conditions that are likely to influence attention, and it systematically varied SOA to explore masking functions. In the Weisstein (1966) study, the target was a letter (D or O) and the observer's task was to report which letter was presented. The mask was a ring that surrounded the contours of the target. The target and mask pair was presented in one of four or one of eight possible locations around a central fixation point. The number of possible locations of the target-mask pair was a variable that was blocked within a session, and the observer knew the number and possible locations. Fig. 10 plots the percent correct detections as a function of ISI, with separate curves for the 4 and 8 possible position conditions. (These data are subsets of the results from experiment two in Weisstein (1966), which were an investigation of serial versus parallel processing rather than a direct study of attentive effects. The other data in the experiment were run in separate sessions, so a comparison of only these curves is valid.)

When there was a difference, masking was stronger in the 8 position condition than in the 4 position condition. Presumably, attention is distributed across the possible positions of the target. With more possible positions, the amount of attention at any given position must be reduced, and therefore identification of the target is more difficult. The masking curve for the 8 position condition has an ISI for maximal 


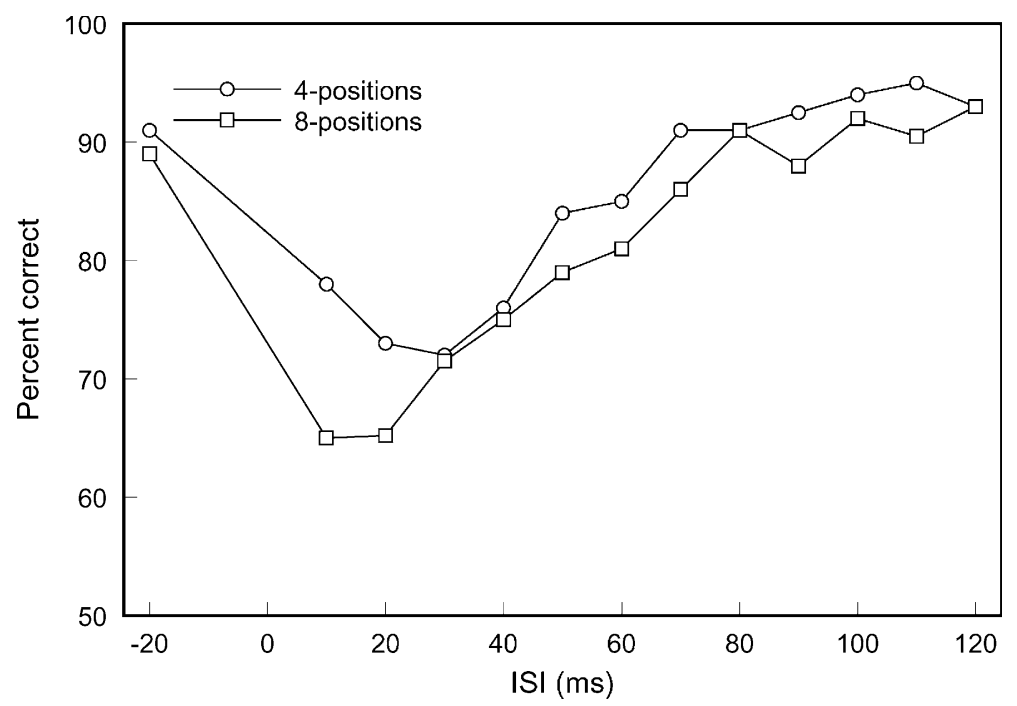

Fig. 10. Experimental data that demonstrate effects of attentional focus on masking functions. With eight possible positions of the target, masking is stronger than for the condition with four possible positions of the target. Moreover, with more positions of the target, the SOA that produces maximum masking (the bottom of the curve) is shifted to a smaller value. (Reproduced from a figure in Weisstein, 1966.)

masking shifted to a smaller value than for the 4 position condition. In efficient masking this combination of changes indicates that the effect of going from the 4 position condition to the 8 position condition is to either strengthen the mask signal or weaken the target signal. That is, having more possible positions where the target may appear not only makes the identification task harder, but it changes the target or mask signal.

Because of the shift in the bottom of the masking function, an efficient masking model cannot make the assumption that attentional focus only changes the mapping of percept strength to behavior. In efficient masking, attention is changing the target or mask signals.

It should be mentioned that other studies that ostensibly also vary attention in a backward masking experiment find different results than Weisstein (1966). For example, Shelley-Tremblay and Mack (1999) explored masking functions for targets that have different tendencies to capture attention (e.g., a happy face versus an inverted face; the observer's name versus another word). They found that differences in targets resulted in differences in clarity ratings and number of detections of letters (in words); with those targets that tend to capture attention showing less masking. Significantly, the strongest masking always occurred for an SOA of $20 \mathrm{~ms}$. Changes in the strength of masking did not result in shifts of the bottom of the masking function. In an efficient masking model, such a property suggests that in the Shelley-Tremblay and Mack (1999) study, variation in attention affected the mapping of percept strength to behavior and did not vary the strength of the target or mask signals. 
Thus, an efficient masking theory would need to make different assumptions about the effect of attention in different experimental conditions. This may prove to be a useful technique for investigating different modes of attention. Perhaps different attentional mechanisms are involved for spatial attention (as in the Weisstein (1966) study) and semantic processing (as in the Shelley-Tremblay \& Mack (1999) study).

Whether this view will prove useful depends in part on a replication of the ShelleyTremblay and Mack (1999) study with a finer-grained measure of the masking function. Shelly-Tremblay and Mack used only SOAS of 0, 20, 60, 100, and 136. Thus, the spacing of the SOA with maximal masking (SOA $=20 \mathrm{~ms}$ ) is a full $40 \mathrm{~ms}$ away from the next largest SOA. The Weisstein (1966) study found a shift of only $20 \mathrm{~ms}$ across different attention conditions. Thus, it is possible that a more precise measure of the masking function in the conditions used by Shelly-Tremblay and Mack will reveal a shift.

\subsection{Increases in masking strength with shifts to larger ISIS}

This section considers a data set that exhibits increases in the overall strength of masking and has corresponding shifts in the bottom of the masking function to larger ISIs. A model that uses efficient masking interprets this finding as evidence that the experimental manipulation that underlies the change in masking strength is varying some component of the mapping between percepts and behavior and is simultaneously varying some component of the target or mask input. This simultaneous impact is necessary for efficient masking to produce these kinds of masking functions. A review of masking literature suggests that this relationship among masking functions within a single study is rare. This lack of data may indicate that people have generally found it difficult to identify situations that produce masking functions that generate this relationship. The lack of data may also suggest that this is an area that deserves more research.

\subsubsection{Word superiority effect}

Michaels and Turvey (1979, experiment E1) compared masking functions produced when the target and mask were three letter words or consonant trigrams. The observer's task was to report as many of the six presented letters as possible. Scoring was based only on the target letters that were correctly reported. Fig. 11 shows data averaged across eye presentation and four observers.

It made little difference whether the mask was a word or a consonant trigram. However, when the target was a word, the masking curve rose after an SOA of $20 \mathrm{~ms}$. In contrast, when the target was a consonant trigram, the masking curves continued to drop to a bottom at an SOA of $40 \mathrm{~ms}$.

A model based on efficient masking would interpret the experimental data as evidence that the difference between the target as a word or non-word has two effects. First, it either decreases the strength of the target signal or increases the strength of the mask signal. This effect is necessary to insure that the bottom of the masking function for a target word is at a smaller SOA than for a non-word target. Second, 


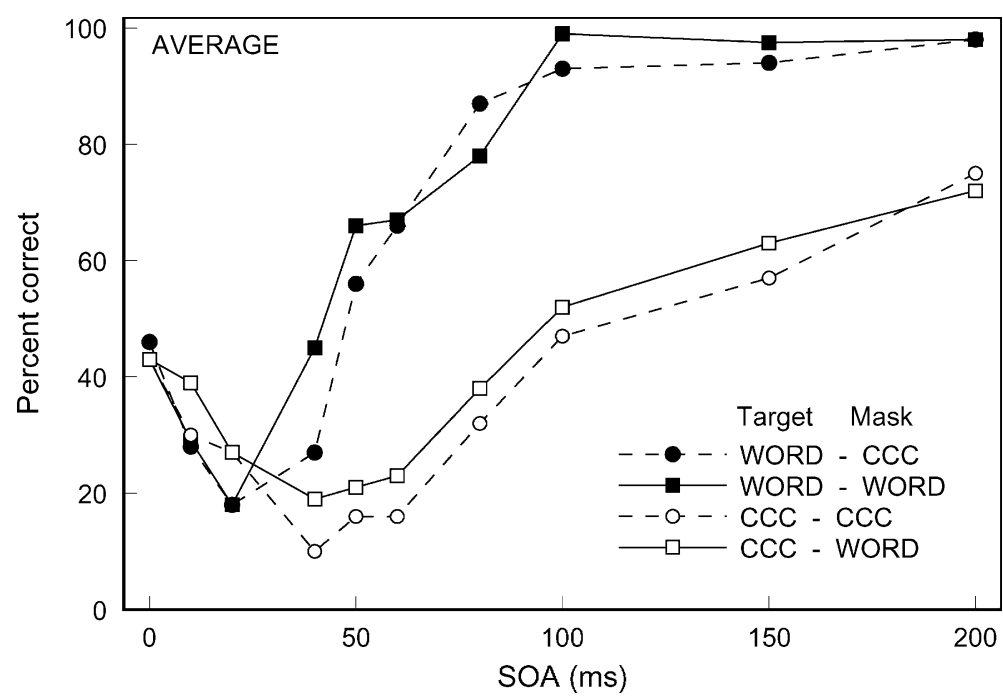

Fig. 11. Experimental data that demonstrate effects of the target and mask being words or non-words. Masking is unchanged with variation in the mask, but changes dramatically when the target is a word versus a non-word. For the target as a word condition, the bottom of the masking function appears at a smaller SOA. (Reproduced from a figure in Michaels \& Turvey, 1979.)

it varies the mapping between the target percept strength and behavior (identification of the letter string) so that, for a given percept strength value, it is easier to identify the characters in a word than in a non-word. The variation in mapping will compensate for the generally stronger masking that would otherwise appear from variation in the target or mask signal (see Fig. 5).

It is less clear what types of mechanisms would be involved in producing these types of effects. The hypothesized change in the mapping of percepts to behavior seems reasonable, as there is a well-known word superiority effect (Reicher, 1969; Wheeler, 1970), whereby detection of letters is easier when the letters are embedded in a word rather than a non-word. The hypothesized decrease in the strength of the target signal and the hypothesized increase in the strength of mask seems more difficult to justify. One could, however, create a number of explanations involving attentional focus or processing times. It could be, for example, that automatic processing of words results in less attention being focused when the target is a word. In the context of backward masking, perhaps this lack of attentional focus results in the target signal being weaker for a word than for a nonword.

Rather than challenge the model by seeing if there are plausible mechanisms for instantiating its functional properties, the model can also be challenged directly at the functional level. An efficient masking model predicts that the data seen in Fig. 11 are the result of a coincidence of two factors. One factor (the variation in the target or mask signal) shifts the bottom of the masking function to a smaller SOA value. The second factor (the variation in the mapping between percepts and 
behavior) shifts the masking function upward. The particular data here seem to have balanced these two effects so that the masking curves for the target word and nonword situations overlap across SOAs less than $20 \mathrm{~ms}$. A model prediction is that those effects will not always balance so nicely and that sometimes the target word masking function will be well below the target non-word masking function, or vice versa. Indeed, the individual data reported by Michaels and Turvey (1979) show exactly this property. Whether this variation across subjects is due to random fluctuations or is indicative of the coincidental nature of the average data will require additional experimentation.

It should be emphasized that the explanation of the data put forth by an efficient masking model is quite different from the one proposed by Michaels and Turvey (1979). They proposed that the branching off of the target word masking functions indicated differences in central processes that differ between words and non-words. In this explanation of the effect, central processes for a target word apparently take over sooner than central processes for a target non-word.

\section{Conclusions}

We have identified basic properties of masking functions produced by a system based on efficient masking and demonstrated how these properties can be used to interpret experimental data in terms of an efficient masking model. This analysis extends the findings in Francis (2000) by further developing an account of ushaped backward masking functions that has not previously been part of any model. Critical to this analysis are shifts in the SOA or ISI that leads to the strongest masking.

To summarize the main findings, in the framework of efficient masking, effects of practice and eccentricity seem to be related to changes in the mapping of percept strength to behavior. Changes in light adaptation, flicker adaptation, and attention seem to be related to changes to either the target or the mask signal. A word superiority effect in masking seems to be related to changes in either the target or the mask signal and to changes in the mapping of percept strength to behavior.

As a model based on efficient masking is further developed, it is important to relate appropriate experimental data to mechanisms. In particular, robust experimental phenomena that are found in many experimental situations (e.g., no shift in the ISI for strongest masking as eccentricity is varied) should be accounted for by general properties of the model. On the other hand, experimental results that are sensitive to conditions and experimental tasks (e.g., the effects of light adaptation) should be accounted for by details of the model (e.g., specific parameter settings or how the general properties of the theory are physically instantiated).

Another example of a sensitive result is the effect of spatial separation between the target and mask in metacontrast masking. Many studies have found that masking weakens as the target and mask are separated, and this phenomenon has been interpreted in several models as due to effects of lateral inhibition (e.g., Bridgeman, 1971; 
Francis, 1997), which would imply that larger separations produce a weaker mask signal. In a model based on efficient masking, the lateral inhibition hypothesis would predict that with increasing separation between the target and the mask, the ISI that produces strongest masking should shift to larger values. There are some experimental data that show this effect (e.g., Growney \& Weisstein, 1972; Growney, 1978; Kolers \& Rosner, 1960; Vrolijk \& van der Wildt, 1985). However, there are also experimental data that find no shift in the ISI that produces strongest masking (e.g., Growney, Weisstein, \& Cox, 1977; Weisstein \& Growney, 1969). It is not clear what differences between the experiments account for the differences in the data properties. Indeed, in Growney (1978) and Weisstein and Growney (1969) different effects of spatial separation are observed for different experiments and/or different observers. The inconsistency across the data suggests that the effect of spatial separation on the shape of the masking function is sensitive to detailed properties of the experimental task and/or the observer. Since the relationship between spatial separation and the shape of the masking function does not seem to be robust across observers and experimental tasks, it is not expected that the general properties of efficient masking would be able to account for the relationship. The properties of efficient masking would only be expected to account for robust properties of the data. This means, for example, that a model based on efficient masking will need to include some specific mechanisms that go beyond hypothesizing that the mask signal simply gets weaker as the target and mask are spatially separated. A model based on efficient masking with that hypothesis would predict that there would be a robust shift in the bottom of the masking function. Since that shift is not always seen, the model must be developed to include other factors as well. Identification of those factors will require additional experimental research.

Within the context of a model based on efficient masking, changes to the $\mathrm{u}$ shaped masking function can be used to identify different aspects of information processing. Backward masking is often used in cognitive psychology as a tool to halt processing of information. The goal is often to identify the time course of information processing, so a strong mask is used to insure that performance on some task increases with the SOA of the target and the mask. Improved performance is taken to indicate further processing, and the SOA at which performance reaches some criterion level can be interpreted as an estimate of the duration of key cognitive processes. This use of masking inherently requires a masking function that is monotonic increasing with SOA. However, the current analysis suggests that in some cases it may also be informative to use a mask that produces a u-shaped masking function. By watching how the bottom of the u-shaped masking function changes with variation in the target, mask, or context it is possible to deduce properties of the system involved in masking.

The analysis given in this paper demonstrates that a system based on efficient masking can be used to account for quite a bit of experimental data. Moreover, the account of the data is fundamentally distinct from the accounts given by other quantitative models of backward masking. Future research needs to identify differences between model types and run experiments to test between the model types. 
It should also be emphasized that the development of a model based on efficient masking remains fairly abstract. The data sets considered here largely match the general properties of the efficient masking theory. As a result, these data sets do not strongly constrain the model in a way that reveals many specific model mechanisms. One implication of this finding is that the general properties of efficient masking can be integrated into a variety of models. In combination with such models, which are possibly constrained by entirely different data sets, it should be possible to generate novel and testable predictions. Even without such generation, the model developed here suggests novel interpretations of existing experimental data and unexpected predictions of future data.

\section{Appendix A. Proofs}

Lemma A.1 (Varying target intensity). For the system defined in Eqs. (1)-(6), the ISI for strongest masking, $\tau_{2}^{*}$, shifts to larger values as target intensity increases

$$
\frac{\partial \tau_{2}^{*}}{\partial I}>0
$$

Proof. In Eq. (6), only the first logarithm contains the term $I$, so the derivative of the other terms will equal zero. If we expand the first logarithm out, we get

$$
\frac{\partial \tau_{2}^{*}}{\partial I}=\frac{\partial}{\partial I} \frac{1}{A}\left[\ln (B I)-\ln (A+C I)+\ln \left(1-\mathrm{e}^{-(A+C I) \tau_{1}}\right)\right] .
$$

Taking the derivative of each term with respect to $I$, we find

$$
\frac{\partial \tau_{2}^{*}}{\partial I}=\frac{1}{A}\left[\frac{1}{I}-\frac{C}{A+C I}+\frac{C \tau_{1} \mathrm{e}^{-(A+C I) \tau_{1}}}{1-\mathrm{e}^{-(A+C I) \tau_{1}}}\right] .
$$

The first term in the brackets is always larger than the second term, so their difference is always positive. The third term is also always positive. So, the entire differential is always greater than zero.

Lemma A.2 (Varying target duration). For the system defined in Eqs. (1)-(6), the ISI for strongest masking, $\tau_{2}^{*}$, shifts to larger values as target duration increases

$$
\frac{\partial \tau_{2}^{*}}{\partial \tau_{1}}>0 .
$$

Proof. The proof is basically the same as for Lemma A.1. After expanding the first logarithm in Eq. (6), only the third term in Eq. (A.2) will vary with $\tau_{1}$. Thus taking the derivative with respect to $\tau_{1}$ will give

$$
\frac{\partial \tau_{2}^{*}}{\partial \tau_{1}}=\frac{A+C I}{A} \frac{\mathrm{e}^{-(A+C I) \tau_{1}}}{1-\mathrm{e}^{-(A+C I) \tau_{1}}} .
$$

This will always be positive.

Corollary A.1 (Saturation of target intensity and duration effects). As target intensity or duration increases, changes in the ISI for maximal masking due to changes in mask intensity and duration will saturate. Mathematically, this means that as I or $\tau_{1}$ increases, $\tau_{2}^{*}$ will not go above a finite upper bound.

Proof. We can calculate the limits directly by watching how Eq. (6) changes with increases in $I$ or $\tau_{1}$. For the limit with respect to $I$, only the first logarithm in Eq. (6) varies with $I$. As $I$ gets larger, the exponential term will go to zero. Likewise, the term $B I /(A+C I)$ goes to the value $B / C$. So 


$$
\lim _{I \rightarrow \infty} \tau_{2}^{*}=\frac{1}{A}\left[\ln \left\{\frac{B}{C}\right\}-\ln \left\{G+\frac{D J\left(1-\mathrm{e}^{-(A+E J) \tau_{3}}\right)}{A+E J}\right\}-(A+E J) \tau_{3}\right],
$$

which is a finite number.

Likewise, for the limit with respect to $\tau_{1}$, only the first logarithm in Eq. (6) varies with $\tau_{1}$. As $\tau_{1}$ increases, the exponential term goes to zero, so

$$
\lim _{\tau_{1} \rightarrow \infty} \tau_{2}^{*}=\frac{1}{A}\left[\ln \left\{\frac{B I}{A+C I}\right\}-\ln \left\{G+\frac{D J\left(1-\mathrm{e}^{-(A+E J) \tau_{3}}\right)}{A+E J}\right\}-(A+E J) \tau_{3}\right],
$$

which is a finite number.

\section{Appendix B. Simulations}

All calculations of percept strength in Figs. 2-5 were based on analytical solutions of $P$ using Eqs. (1)-(5). The percept strength, $P$, is the duration of the target VRF above threshold, and it can be written as

$$
P=T_{\text {off }}-T_{\text {on }}
$$

Here, $T_{\text {off }}$ corresponds to the time at which the VRF equals the threshold after the target has turned off (e.g., $x(t)=G$, for $t>\tau_{1}$ ). $T_{\text {on }}$ corresponds to the time at which the VRF equals the threshold during target presentation (e.g., $x(t)=G$ for $0<t \leqslant \tau_{1}$ ).

The solution for $x(t)$ was reported in Francis (2000), so computing $T_{\text {on }}$ becomes a matter of algebra, and yields

$$
T_{\text {on }}=-\frac{\ln [1-G(A+C I) /(B I)]}{A+C I} .
$$

The parameters $A, B, C, G$, and $I$ where always chosen so that $T_{\text {on }}$ was a positive value. If the VRF never went about the value $G$, the equation would be undefined (and there would be no percept).

Computing $T_{\text {off }}$ requires consideration of the possible ways the VRF could decay to the threshold. It could reach the threshold during the ISI, before the mask appears. It could reach the threshold during the mask presentation. Or it could reach the threshold after the mask offset. The solution in each case is derived by applying algebra to the derivation of $x(t)$ in Francis (2000). The result is as follows:

$$
T_{\text {off }}=\left\{\begin{array}{l}
\tau_{1}+\frac{1}{A} \ln \left[\frac{x\left(\tau_{1}\right)}{G}\right] \quad \text { for } x\left(\tau_{1}\right)>G \text { and } x\left(\tau_{1}+\tau_{2}\right) \leqslant G, \\
\tau_{1}+\tau_{2}+\frac{1}{A+E J} \ln \left[\frac{x\left(\tau_{1}+\tau_{2}\right)+D J /(A+E J)}{G+D J /(A+E J)}\right] \quad \text { for } x\left(\tau_{1}+\tau_{2}\right)>G \text { and } x\left(\tau_{1}+\tau_{2}+\tau_{3}\right) \leqslant G, \\
\tau_{1}+\tau_{2}+\tau_{3}+\frac{1}{A} \ln \left[\frac{x\left(\tau_{1}+\tau_{2}+\tau_{3}\right)}{G}\right] \quad \text { for } x\left(\tau_{1}+\tau_{2}+\tau_{3}\right)>G .
\end{array}\right.
$$

Unless otherwise indicated, all simulations used the following parameters: $A=0.01, B=1.0, C=1.0$, $D=0.001, E=0.001, G=0.2, I=10, J=10, \tau_{1}=15$, and $\tau_{3}=15$.

For the computation of hypothetical masking functions in Figs. $4 \mathrm{~b}$ and $5 \mathrm{~b}$, percept strength $P$ was combined with the ceiling, $H_{\mathrm{c}}$, and floor, $H_{\mathrm{f}}$, thresholds to produce a number between 0 and 100 . The calculation was

$$
K=100 \times \min \left[\frac{\max \left[P-H_{\mathrm{f}}, 0\right]}{H_{\mathrm{c}}-H_{\mathrm{f}}}, 1.0\right]
$$

which insures that any $P$ value less than the floor threshold produces a $K$ value of zero, any $P$ value bigger than the ceiling threshold produces a $K$ value of 100, and other $P$ values produce $K$ values that are linearly related to their relative position between the floor and ceiling thresholds. The values of $H_{\mathrm{c}}$ and $H_{\mathrm{f}}$ can be read off the graph in Fig. 4a and the text discussing Fig. 5. 


\section{References}

Alpern, M. (1953). Metacontrast. Journal of the Optical Society of America, 43, 648-657.

Anstis, S. M. (1974). A chart demonstrating variations in acuity with retinal position. Vision Research, 14, 589-592.

Bernstein, I. H., \& Fisicaro, S. A. (1977). Effects of absolute mask energy and mask energy range on metacontrast suppression. Perception \& Psychophysics, 22, 359-365.

Bischof, W. F., \& Di Lollo, V. (1995). Motion and metacontrast with simultaneous onset of stimuli. Journal of the Optical Society of America, A, 12, 1623-1636.

Breitmeyer, B. (1978). Metacontrast masking as a function of mask energy. Bulletin of the Psychonomic Society, 12, 50-52.

Breitmeyer, B. (1984). Visual masking: An integrative approach. New York: Oxford University Press.

Breitmeyer, B., \& Ganz, L. (1976). Implications of sustained and transient channels for theories of visual pattern masking, saccadic suppression, and information processing. Psychological Review, 83, $1-36$.

Breitmeyer, B., \& Ögmen, H. (2000). Recent models and findings in visual backward masking: A comparison, review, and update. Perception \& Psychophysics, 62, 1572-1595.

Bridgeman, B. (1971). Metacontrast and lateral inhibition. Psychological Review, 78, 528-539.

Bridgeman, B., \& Leff, S. (1979). Interaction of stimulus size and retinal eccentricity in metacontrast masking. Journal of Experimental Psychology: Human Perception and Performance, 1, 101-109.

Busey, T., \& Loftus, G. (1994). Sensory and cognitive components of visual information acquisition. Psychological Review, 101, 446-469.

Dosher, B., \& Lu, Z.-L. (1998). Perceptual learning reflects external noise filtering and internal noise reduction through channel reweighting. Proceedings of the National Academy of Science, 95, 1398813993.

Enns, J. T., \& Di Lollo, V. (1997). Object substitution: A new form of masking in unattended visual locations. Psychological Science, 8, 135-139.

Francis, G. (1997). Cortical dynamics of lateral inhibition: Metacontrast masking. Psychological Review, 104, 572-594.

Francis, G. (1999). Spatial frequency and visual persistence: Cortical reset. Spatial Vision, 12, 31-50.

Francis, G. (2000). Quantitative theories of metacontrast masking. Psychological Review, 107, 768-785.

Gardner, M. (1961). The 2nd scientific American book of mathematical puzzles \& diversions. New York: Simon \& Schuster.

Gaudiano, P. (1992). A unified neural model of spatiotemporal processing in $X$ and $Y$ retinal ganglion cells. Biological Cybernetics, 67, 11-21.

Gold, J., Bennett, P. J., \& Sekuler, A. B. (1999). Signal but not noise changes with perceptual learning. Nature, 402, 176-178.

Grossberg, S. (1983). The quantized geometry of visual space: The coherent computation of depth, form and lightness. The Behavioral and Brain Sciences, 6, 625-657.

Growney, R. (1978). Metacontrast as a function of the spatial frequency composition of the target and mask. Vision Research, 18, 1117-1123.

Growney, R., \& Weisstein, N. (1972). Spatial characteristics of metacontrast. Journal of the Optical Society of America, 62, 690-696.

Growney, R., Weisstein, N., \& Cox, S. (1977). Metacontrast as a function of spatial separation with narrow line targets and masks. Vision Research, 17, 1201-1205.

Hellige, J. B., Walsh, D. A., Lawrence, V. W., \& Prasse, M. (1979). Figural relationship effects and mechanisms of visual masking. Journal of Experimental Psychology: Human Perception and Performance, 5, 88-100.

Hogben, J. H., \& Di Lollo, V. (1984). Practice reduces suppression in metacontrast and in apparent motion. Perception \& Psychophysics, 35, 441-445.

Kolers, P., \& Rosner, B. (1960). On visual masking (metacontrast): Dichoptic observation. American Journal of Psychology, 73, 2-21.

Kolers, P. A. (1962). Intensity and contour effects in visual masking. Vision Research, 2, $277-294$. 
Loftus, G. R., Duncan, J., \& Gehrig, P. (1992). On the time course of perceptual information that results from a brief visual presentation. Journal of Experimental Psychology: Human Perception and Performance, 18, 530-549.

Michaels, C. F., \& Turvey, M. T. (1979). Central sources of visual masking: Indexing structures supporting seeing at a single, brief glance. Psychological Research, 41, 1-61.

Petry, S., Grigonis, A., \& Reichert, B. (1979). Decrease in metacontrast masking following adaptation to flicker. Perception, 8, 541-547.

Purcell, D. G., Stewart, A. L., \& Brunner, R. L. (1974). Metacontrast target detection under light and dark adaptation. Bulletin of the Psychonomic Society, 3, 199-201.

Ramachandran, V. I., \& Cobb, S. (1995). Visual attention modulates metacontrast masking. Nature, 373, $66-68$.

Reicher, G. M. (1969). Perceptual recognition as a function of meaningfulness of stimulus material. Journal of Experimental Psychology, 81, 275-280.

Shelley-Tremblay, J., \& Mack, A. (1999). Metacontrast masking and attention. Psychological Science, 10, 508-515.

Schiller, P. (1965). Metacontrast interference as determined by a method of comparisons. Perceptual and Motor Skills, 20, 279-285.

Spencer, T. J., \& Shuntich, R. (1970). Evidence for an interruption theory of backward masking. Journal of Experimental Psychology, 85, 198-203.

Sperling, G., \& Sondhi, M. (1968). Model for visual luminance discrimination and flicker detection. Journal of the Optical Society of America, 58, 1133-1145.

Stewart, A. L., \& Purcell, D. G. (1970). U-shaped masking functions in visual backward masking: Effects of target configuration and retinal position. Perception \& Psychophysics, 7, 253-256.

Stewart, A. L., \& Purcell, D. G. (1974). Visual backward masking by a flash of light: A study of U-shaped detection functions. Journal of Experimental Psychology, 103, 553-566.

Turvey, M. T. (1973). On peripheral and central processes in vision: Inferences from an information processing analysis of masking with patterned stimuli. Psychological Review, 80, 1-52.

Vrolijk, P. C., \& van der Wildt, G. J. (1985). The influence of the background on the generation of inhibition. Vision Research, 25, 1423-1429.

Weisstein, N. (1966). Backward masking and models of perceptual processing. Journal of Experimental Psychology, 72, 232-240.

Weisstein, N. (1972). Metacontrast. In D. Jameson, \& L. Hurvich (Eds.), Visual psychophysics (Vol. 7(4)). Handbook of sensory physiology. Berlin: Springer.

Weisstein, N., \& Growney, R. L. (1969). Apparent movement and metacontrast: A note on Kahneman's formulation. Perception \& Psychophysics, 5, 321-328.

Werner, H. (1935). Studies on contour: I. Qualitative analyses. American Journal of Psychology, 47, 40-64.

Wheeler, D. D. (1970). Processes in word recognition. Cognitive Psychology, 1, 59-85. 